\title{
Descripción, ampliación y nuevo registro de distribución para Dendropsophus manonegra (Rivera \& Orrico, 2013) y Dendropsophus sarayacuensis (Shreve, 1935) (Amphibia: Anura: Hylidae) en el piedemonte andino-amazónico del departamento de Caquetá, Colombia
}

\author{
Alejandro Navarro-Morales ${ }^{1, *}$, Diego Huseth Ruiz-Valderrama $^{2}$ \\ ${ }^{1}$ Semillero de Investigación en Herpetología - SEH, Universidad de la Amazonia, Florencia, Caquetá, Colombia \\ ${ }^{2}$ Grupo de Fauna silvestre, Centro de Investigación de la Biodiversidad Andino-Amazónica, INBIANAM, \\ Universidad de la Amazonia, Florencia, Caquetá, Colombia
}

\begin{abstract}
Resumen
Se presentan la ampliación y nuevos registros de distribución de dos especies de la familia Hylidae pertenecientes al género Dendropsophus en la región andino-amazónica del departamento del Caquetá, Colombia. Los registros se hicieron en tres localidades del municipio de Florencia: vereda Villaraz, vereda Paraíso y vereda La Holanda, y dos del municipio de Belén de los Andaquíes: Resguardo Indígena La Serinda y vereda Agua Dulce. Utilizando el método de encuentro visual desde las 19:00 a las 23:00 se recolectaron 38 individuos de Dendropsophus manonegra y tres de Dendropsophus sarayacuensis; se hizo la descripción taxonómica de los individuos en vida y preservados. Se discuten, además, las variaciones altitudinales en las que se pueden encontrar estos organismos a partir de investigaciones de diferentes autores. Estos nuevos registros son fundamentales para el conocimiento de la herpetofauna colombiana, en especial la del departamento del Caquetá.
\end{abstract}

Palabras clave: Dendropsophus; Distribución; Piedemonte andino-amazónico; Caquetá.

Description, extension, and new distribution register for Dendropsophus manonegra (Rivera \& Orrico, 2013) and Dendropsophus sarayacuensis (Shreve, 1935) (Amphibia: Anura: Hylidae) in the Andean-Amazon piedmont region in the department of Caquetá - Colombia

\begin{abstract}
We report the extension of the present distribution area, as well as new records for two species of the genus Dendropsophus, family Hylidae, in the Andean-Amazonian region of the department of Caquetá, Colombia: In three localities of the municipality of Florencia: Villaraz, Paraíso, La Holanda, and two in the municipality of Belén de los Andaquíes: the indigenous reservation La Serinda and Agua Dulce. By visual encounter from 19:00 - 23:00, we collected 38 individuals of Dendropsophus manonegra and three of Dendropsophus sarayacuensis; we made the taxonomic description of the individuals while alive and already preserved. We also discuss here the altitudinal variations in which these species are usually found as reported in studies by different authors. The new records are fundamental for the knowledge of the Colombian herpetofauna, especially in the department of Caquetá.
\end{abstract}

Key words: Dendropsophus; Distribution; Andean-Amazonian piedmont; Caquetá.

\section{Introducción}

La familia Hylidae posee una gran diversidad de especies distribuidas en el Neotrópico y se encuentra en una gran variedad de microhábitats dentro de los ecosistemas (Lynch \& Suarez, 2011). En Colombia se han registrado 127 especies distribuidas en 13 géneros (Frost, 2019), siendo Dendropsophus uno de los más diversos en términos de abundancia y riqueza (Duellman, et al., 2016): 35 especies que corresponden al 27,5\% de los hílidos en Colombia (Acosta-Galvis, 2019).
Dendropsophus manonegra es una especie recientemente descrita por Rivera-Correa \& Orrico (2013), con distribución exclusiva en los departamentos colombianos de Cauca, Caquetá y Putumayo, entre los 400 y los 1.200 (m s.n.m.) (Rivera-Correa \& Orrico, 2013).

\footnotetext{
*Correspondencia:

Alejandro Navarro-Morales; e-mail: alejanura@hotmail.com

Recibido: 8 de mayo de 2019

Aceptado: 10 de julio de 2019

Editor: Jaime Cantera
} 
Dendropsophus sarayacuensis fue descrita por Shreve (1935) y se encuentra distribuida en Bolivia, Brasil, Colombia, Ecuador, Perú y Venezuela (La Marca, et al., 2004); en Colombia se registra en los departamentos de Caquetá, Vaupés y Amazonas, entre los 100 y 1.000 m s.n.m. (Cochran \& Goin, 1970; Acosta-Galvis, 2000; Lynch, 2005; Lynch, 2007; Lynch \& Suarez, 2011; Osorno, et al., 2011).

Estas especies son de hábitos nocturnos y se encuentran en charcas temporales o permanentes y en áreas abiertas o con cobertura vegetal (Ramírez \& Duellman, 1994); sus procesos de reproducción se asocian con la temporada de lluvias abundantes (Aichinger, 1987) y sus ovoposiciones se hacen sobre raíces, troncos y hojas cercanas al espejo de agua (Hödl, 1991), características que evidencian la simpatría de especies.

Dendropsophus manonegra, D. bifurcus y D. sarayacuensis son especies hermanas que se agrupan dentro del complejo de especies Dendropsophus leucophyllatus, (Rivera-Correa \& Orrico, 2013) y comparten características morfológicas como el cuerpo ancho, una membrana axilar desarrollada y una coloración que varía de amarillo a rojo en membranas y regiones ocultas (axilas, ingle, pantorrillas) (Faivovich, et al., 2005; Jungfer, et al., 2010). Aunque D. manonegra es una excepción, ya que presenta una coloración negro-azulada (Rivera-Correa \& Orrico, 2013), las características moleculares permiten corroborar su agrupación dentro del complejo (Fouquet, et al., 2007; Caminer, et al., 2017).

En este contexto, en el presente estudio se amplía el rango de distribución y se reporta de un nuevo registro de distribución para $D$. manonegra y D. sarayacuensis en el piedemonte andino-amazónico de departamento de Caquetá, Colombia.

\section{Metodología}

Se utilizó el método de búsqueda libre y sin restricción por encuentro visual y captura directa en la vegetación asociada con charcas permanentes o temporales entre las 19:00 y las 23:00. D. manonegra se registró y se recolectó en cuatro localidades de Caquetá: en el municipio de Belén de los Andaquíes, veredas Pueblo Nuevo y Los Ángeles, y en el Resguardo Indígena La Serinda; en el municipio de Florencia, corregimiento el Caraño, veredas Villaraz y Paraíso, corregimiento de Santo Domingo, vereda La Holanda, y Reserva Natural y Ecoturística La Avispa, en tanto que $D$. sarayacuensis se registró y se recolectó en el municipio de Belén de los Andaquíes, en la vereda Agua Dulce, finca Las Brisas (Figura 1; Tabla 1).

\section{Resultados}

Se recolectaron 38 individuos pertenecientes a $D$. manonegra; en el municipio de Florencia, vereda Villaraz, se encontraron a una distancia de $8,06 \mathrm{~km}$ en línea recta de la localidad tipo; en la vereda Paraíso, a una distancia de 5,75 km en línea recta de la localidad tipo; en la vereda La Holanda y en la Reserva Natural y Ecoturística La Avisa, a 19,61 Km en línea recta de la localidad tipo, y en el municipio de Belén de los Andaquíes y el Resguardo Indígena La Serinda, a una distancia de $32,10 \mathrm{~km}$ en línea recta de la localidad tipo (Figura 1; Tabla 1).

Se recolectaron tres especímenes de D. sarayacuensis en el municipio de Belén de los Andaquíes, vereda Agua Dulce, a $542,66 \mathrm{~km}$ de la localidad tipo y a $55 \mathrm{~km}$ en línea recta del último registro para esta especie en Caquetá (Figura 1; Tabla 1). Los especímenes recolectados se depositaron en la colección de herpetología del Museo de Historia Natural-UAM de la Universidad de la Amazonia (Florencia - Caquetá).

Descripción de ejemplares Dendropsophus manonegra. Estos especímenes presentan cabeza ancha como su cuerpo, ligeramente más larga que ancha; la longitud de los dígitos palmares se observa en la siguiente fórmula $\mathrm{I}<\mathrm{II}<\mathrm{IV}<\mathrm{III}$ (Figura 2A) y los dígitos pediales se observa en la siguiente fórmula I $<$ II $<$ III $<\mathrm{V}<\mathrm{IV}$ (Figura 2B). Presenta un saco bucal único de coloración gris clara; presencia de dos parches glandulares ubicados en la región pectoral separados entre sí, con coloración blanca (Figura 3A); coloración café oscura y puntos negros dispersos, coloración negra brillante en partes ocultas de la región de la pantorrilla y membranas interdigitales pediales y manuales; línea bifurcada completa en la región cefálica y dorsolateral que comprenden hasta la ingle con mancha en el talón de color crema (Figura 3B).

Los ejemplares de $D$. manonegra vivos tienen el dorso café claro, con puntos de color negro dispersos; los flancos y los miembros anteriores y posteriores son de coloración marrón oscuro (Figura 4A); las regiones ocultas de las extremidades presentan una coloración negra, al igual

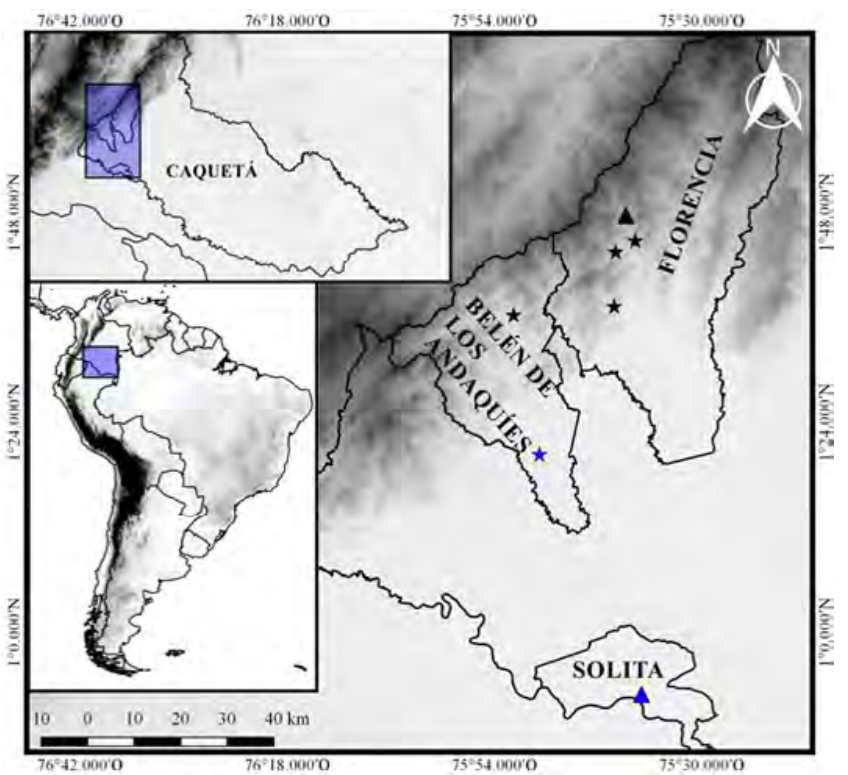

Figura 1. Registros de distribución para Dendropsophus manonegra y Dendropsophus sarayacuensis. Triangulo negro: antiguo registro; estrella negra: nuevos registros para D. manonegra; triangulo Azul: antiguo registro; estrella azul: nuevo registro para D.sarayacuensis 
Tabla 1. Registros por localidad de ranas del género Dendropsophus (Hylidae) en la región amazónica colombiana. UAM-H: Universidad de la Amazonia - Colección Herpetológica

\begin{tabular}{|c|c|c|c|c|c|}
\hline Especie & $\begin{array}{l}\text { Código } \\
\text { UAM-H }\end{array}$ & Fecha & Localidad & Coordenadas & $\begin{array}{c}\text { Altura } \\
\text { (m s.n.m) }\end{array}$ \\
\hline Dendropsophus manonegra & UAM-H 022 & 2005 & Caquetá, Florencia, Villaraz & $01^{\circ} 43.492^{\prime} \mathrm{N}, 75^{\circ} 39.981^{\prime} \mathrm{O}$ & 850 \\
\hline Dendropsophus manonegra & UAM-H 023 & 2005 & Caquetá, Florencia, Villaraz & $01^{\circ} 43.492^{\prime} \mathrm{N}, 75^{\circ} 39.981^{\prime} \mathrm{O}$ & 850 \\
\hline Dendropsophus manonegra & UAM-H 032 & 2005 & Caquetá, Florencia, Villaraz & $01^{\circ} 43.492^{\prime} \mathrm{N}, 75^{\circ} 39.981^{\prime} \mathrm{O}$ & 850 \\
\hline Dendropsophus manonegra & UAM-H 616 & 2005 & Caquetá, Florencia, Villaraz & $01^{\circ} 43.492^{\prime} \mathrm{N}, 75^{\circ} 39.981^{\prime} \mathrm{O}$ & 850 \\
\hline Dendropsophus manonegra & UAM-H 617 & 2005 & Caquetá, Florencia, Villaraz & $01^{\circ} 43.492^{\prime} \mathrm{N}, 75^{\circ} 39.981^{\prime} \mathrm{O}$ & 850 \\
\hline Dendropsophus manonegra & UAM-H 618 & 2005 & Caquetá, Florencia, Villaraz & $01^{\circ} 43.492^{\prime} \mathrm{N}, 7^{\circ} 39.981^{\prime} \mathrm{O}$ & 850 \\
\hline Dendropsophus manonegra & UAM-H 619 & 2005 & Caquetá, Florencia, Villaraz & $01^{\circ} 43.492^{\prime} \mathrm{N}, 75^{\circ} 39.981^{\prime} \mathrm{O}$ & 850 \\
\hline Dendropsophus manonegra & UAM-H 620 & 2005 & Caquetá, Florencia, Villaraz & $01^{\circ} 43.492^{\prime} \mathrm{N}, 75^{\circ} 39.981^{\prime} \mathrm{O}$ & 850 \\
\hline Dendropsophus manonegra & UAM-H 621 & 2005 & Caquetá, Florencia, Villaraz & $01^{\circ} 43.492^{\prime} \mathrm{N}, 75^{\circ} 39.981^{\prime} \mathrm{O}$ & 850 \\
\hline Dendropsophus manonegra & UAM-H 622 & 2005 & Caquetá, Florencia, Villaraz & $01^{\circ} 43.492^{\prime} \mathrm{N}, 75^{\circ} 39.981^{\prime} \mathrm{O}$ & 850 \\
\hline Dendropsophus manonegra & UAM-H 623 & 2005 & Caquetá, Florencia, Villaraz & $01^{\circ} 43.492^{\prime} \mathrm{N}, 75^{\circ} 39.981^{\prime} \mathrm{O}$ & 850 \\
\hline Dendropsophus manonegra & UAM-H 989 & 2007 & $\begin{array}{l}\text { Caquetá, Belén de los Andaquíes, } \\
\text { Resguardo Indígena La Serinda }\end{array}$ & $01^{\circ} 36^{\prime} 08.6^{\prime \prime} \mathrm{N}, 75^{\circ} 51^{\prime} 49.1^{\prime \prime} \mathrm{O}$ & 470 \\
\hline Dendropsophus manonegra & UAM-H 990 & 2007 & $\begin{array}{l}\text { Caquetá, Belén de los Andaquíes, } \\
\text { Resguardo Indígena La Serinda }\end{array}$ & $01^{\circ} 36^{\prime} 08.6^{\prime \prime} \mathrm{N}, 75^{\circ} 51^{\prime} 49.1^{\prime \prime} \mathrm{O}$ & 470 \\
\hline Dendropsophus manonegra & UAM-H 991 & 2007 & $\begin{array}{l}\text { Caquetá, Belén de los Andaquíes, } \\
\text { Resguardo Indígena La Serinda }\end{array}$ & $01^{\circ} 36^{\prime} 08.6^{\prime \prime} \mathrm{N}, 75^{\circ} 51^{\prime} 49.1^{\prime \prime} \mathrm{O}$ & 470 \\
\hline Dendropsophus manonegra & UAM-H 992 & 2007 & $\begin{array}{l}\text { Caquetá, Belén de los Andaquíes, } \\
\text { Resguardo Indígena La Serinda }\end{array}$ & $01^{\circ} 36^{\prime} 08.6^{\prime \prime} \mathrm{N}, 75^{\circ} 51^{\prime} 49.1^{\prime \prime} \mathrm{O}$ & 470 \\
\hline Dendropsophus manonegra & UAM-H 993 & 2007 & $\begin{array}{l}\text { Caquetá, Belén de los Andaquíes, } \\
\text { Resguardo Indígena La Serinda }\end{array}$ & $01^{\circ} 36^{\prime} 08.6^{\prime \prime} \mathrm{N}, 75^{\circ} 51^{\prime} 49.1^{\prime \prime} \mathrm{O}$ & 470 \\
\hline Dendropsophus manonegra & UAM-H 994 & 2007 & $\begin{array}{l}\text { Caquetá, Belén de los Andaquíes, } \\
\text { Resguardo Indígena La Serinda }\end{array}$ & $01^{\circ} 36^{\prime} 08.6^{\prime \prime} \mathrm{N}, 75^{\circ} 51^{\prime} 49.1^{\prime \prime} \mathrm{O}$ & 470 \\
\hline Dendropsophus manonegra & UAM-H 995 & 2007 & $\begin{array}{l}\text { Caquetá, Belén de los Andaquíes, } \\
\text { Resguardo Indígena La Serinda }\end{array}$ & $01^{\circ} 36^{\prime} 08.6^{\prime \prime} \mathrm{N}, 75^{\circ} 51^{\prime} 49.1^{\prime \prime} \mathrm{O}$ & 470 \\
\hline Dendropsophus manonegra & UAM-H 996 & 2007 & $\begin{array}{l}\text { Caquetá, Belén de los Andaquíes, } \\
\text { Resguardo Indígena La Serinda }\end{array}$ & $01^{\circ} 36^{\prime} 08.6^{\prime \prime} \mathrm{N}, 75^{\circ} 51^{\prime} 49.1^{\prime \prime} \mathrm{O}$ & 470 \\
\hline Dendropsophus manonegra & UAM-H 997 & 2007 & $\begin{array}{l}\text { Caquetá, Belén de los Andaquíes, } \\
\text { Resguardo Indígena La Serinda }\end{array}$ & $01^{\circ} 36^{\prime} 08.6^{\prime \prime} \mathrm{N}, 75^{\circ} 51^{\prime} 49.1^{\prime \prime} \mathrm{O}$ & 470 \\
\hline Dendropsophus manonegra & UAM-H 998 & 2007 & $\begin{array}{l}\text { Caquetá, Belén de los Andaquíes, } \\
\text { Resguardo Indígena La Serinda }\end{array}$ & $01^{\circ} 36^{\prime} 08.6^{\prime \prime} \mathrm{N}, 75^{\circ} 51^{\prime} 49.1^{\prime \prime} \mathrm{O}$ & 470 \\
\hline Dendropsophus manonegra & UAM-H 999 & 2007 & $\begin{array}{l}\text { Caquetá, Belén de los Andaquíes, } \\
\text { Resguardo Indígena La Serinda }\end{array}$ & $01^{\circ} 36^{\prime} 08.6^{\prime \prime} \mathrm{N}, 75^{\circ} 51^{\prime} 49.1^{\prime \prime} \mathrm{O}$ & 470 \\
\hline Dendropsophus manonegra & UAM-H 1000 & 2007 & $\begin{array}{l}\text { Caquetá, Belén de los Andaquíes, } \\
\text { Resguardo Indígena La Serinda }\end{array}$ & $01^{\circ} 36^{\prime} 08.6^{\prime \prime} \mathrm{N}, 75^{\circ} 51^{\prime} 49.1^{\prime \prime} \mathrm{O}$ & 470 \\
\hline Dendropsophus manonegra & UAM-H 1001 & 2007 & $\begin{array}{l}\text { Caquetá, Belén de los Andaquíes, } \\
\text { Resguardo Indígena La Serinda }\end{array}$ & $01^{\circ} 36^{\prime} 08.6^{\prime \prime} \mathrm{N}, 75^{\circ} 51^{\prime} 49.1^{\prime \prime} \mathrm{O}$ & 470 \\
\hline Dendropsophus manonegra & UAM-H 1002 & 2007 & $\begin{array}{l}\text { Caquetá, Belén de los Andaquíes, } \\
\text { Resguardo Indígena La Serinda }\end{array}$ & $01^{\circ} 36^{\prime} 08.6^{\prime \prime} \mathrm{N}, 75^{\circ} 51^{\prime} 49.1^{\prime \prime} \mathrm{O}$ & 470 \\
\hline Dendropsophus manonegra & UAM-H 1003 & 2007 & $\begin{array}{l}\text { Caquetá, Belén de los Andaquíes, } \\
\text { Resguardo Indígena La Serinda }\end{array}$ & $01^{\circ} 36^{\prime} 08.6^{\prime \prime} \mathrm{N}, 75^{\circ} 51^{\prime} 49.1^{\prime \prime} \mathrm{O}$ & 470 \\
\hline Dendropsophus manonegra & UAM-H 1004 & 2007 & $\begin{array}{l}\text { Caquetá, Belén de los Andaquíes, } \\
\text { Resguardo Indígena La Serinda }\end{array}$ & $01^{\circ} 36^{\prime} 08.6^{\prime \prime} \mathrm{N}, 75^{\circ} 51^{\prime} 49.1^{\prime \prime} \mathrm{O}$ & 470 \\
\hline Dendropsophus manonegra & UAM-H 1005 & 2007 & $\begin{array}{l}\text { Caquetá, Belén de los Andaquíes, } \\
\text { Resguardo Indígena La Serinda }\end{array}$ & $01^{\circ} 36^{\prime} 08.6^{\prime \prime} \mathrm{N}, 75^{\circ} 51^{\prime} 49.1^{\prime \prime} \mathrm{O}$ & 470 \\
\hline Dendropsophus manonegra & UAM-H 1007 & 2007 & $\begin{array}{l}\text { Caquetá, Belén de los Andaquíes, } \\
\text { Resguardo Indígena La Serinda }\end{array}$ & $01^{\circ} 36^{\prime} 08.6^{\prime \prime} \mathrm{N}, 75^{\circ} 51^{\prime} 49.1^{\prime \prime} \mathrm{O}$ & 470 \\
\hline Dendropsophus manonegra & UAM-H 1008 & 2007 & $\begin{array}{l}\text { Caquetá, Belén de los Andaquíes, } \\
\text { Resguardo Indígena La Serinda }\end{array}$ & $01^{\circ} 36^{\prime} 08.6^{\prime \prime} \mathrm{N}, 75^{\circ} 51^{\prime} 49.1^{\prime \prime} \mathrm{O}$ & 470 \\
\hline Dendropsophus manonegra & UAM-H 1009 & 2007 & $\begin{array}{l}\text { Caquetá, Belén de los Andaquíes, } \\
\text { Resguardo Indígena La Serinda }\end{array}$ & $01^{\circ} 36^{\prime} 08.6^{\prime \prime} \mathrm{N}, 75^{\circ} 51^{\prime} 49.1^{\prime \prime} \mathrm{O}$ & 470 \\
\hline Dendropsophus manonegra & UAM-H 1010 & 2007 & $\begin{array}{l}\text { Caquetá, Belén de los Andaquíes, } \\
\text { Resguardo Indígena La Serinda }\end{array}$ & $01^{\circ} 36^{\prime} 08.6^{\prime \prime} \mathrm{N}, 75^{\circ} 51^{\prime} 49.1^{\prime \prime} \mathrm{O}$ & 470 \\
\hline
\end{tabular}


Rev. Acad. Colomb. Cienc. Ex. Fis. Nat. 43(168):502-507, julio-septiembre de 2019 doi: http://dx.doi.org/10.18257/raccefyn.889
Nuevo registro de distribución de especies de Hylidae en el piedemonte andino-amazónico, Caquetá, Colombia

\begin{tabular}{|c|c|c|c|c|c|}
\hline Dendropsophus manonegra & UAM-H 1011 & 2007 & $\begin{array}{l}\text { Caquetá, Belén de los Andaquíes, } \\
\text { Resguardo Indígena La Serinda }\end{array}$ & $01^{\circ} 36^{\prime} 08.6^{\prime \prime} \mathrm{N}, 75^{\circ} 51^{\prime} 49.1^{\prime \prime} \mathrm{O}$ & 470 \\
\hline Dendropsophus manonegra & UAM-H 1012 & 2007 & $\begin{array}{l}\text { Caquetá, Belén de los Andaquíes, } \\
\text { Resguardo Indígena La Serinda }\end{array}$ & $01^{\circ} 36^{\prime} 08.6^{\prime \prime} \mathrm{N}, 75^{\circ} 51^{\prime} 49.1^{\prime \prime} \mathrm{O}$ & 470 \\
\hline Dendropsophus manonegra & UAM-H 1013 & 2007 & $\begin{array}{l}\text { Caquetá, Belén de los Andaquíes, } \\
\text { Resguardo Indígena La Serinda }\end{array}$ & $01^{\circ} 36^{\prime} 08.6^{\prime \prime} \mathrm{N}, 75^{\circ} 51^{\prime} 49.1^{\prime \prime} \mathrm{O}$ & 470 \\
\hline Dendropsophus manonegra & UAM-H 1512 & 2017 & Caquetá, Florencia, Paraíso & $01^{\circ} 44^{\prime} 46.46^{\prime \prime} \mathrm{N}, 75^{\circ} 37^{\prime} 44.24^{\prime \prime} \mathrm{O}$ & 673 \\
\hline Dendropsophus manonegra & UAM-H 1513 & 2017 & Caquetá, Florencia, Paraíso & $01^{\circ} 44^{\prime} 46.46^{\prime \prime} \mathrm{N}, 75^{\circ} 37^{\prime} 44.24^{\prime \prime} \mathrm{O}$ & 673 \\
\hline Dendropsophus manonegra & UAM-H 1514 & 2017 & Caquetá, Florencia, Paraíso & $01^{\circ} 44^{\prime} 46.46^{\prime \prime} \mathrm{N}, 75^{\circ} 37^{\prime} 44.24^{\prime \prime} \mathrm{O}$ & 673 \\
\hline Dendropsophus sarayacuensis & UAM-H 1485 & 2012 & $\begin{array}{l}\text { Caquetá, Belén de los Andaquíes, } \\
\text { Agua Dulce }\end{array}$ & $01^{\circ} 20^{\prime} 19.50 \mathrm{~N}, 75^{\circ} 48^{\prime} 49.35^{\prime \prime} \mathrm{O}$ & 268 \\
\hline Dendropsophus sarayacuensis & UAM-H 1486 & 2012 & $\begin{array}{l}\text { Caquetá, Belén de los Andaquíes, } \\
\text { Agua Dulce }\end{array}$ & $01^{\circ} 20^{\prime} 19.50 \mathrm{~N}, 75^{\circ} 48^{\prime} 49.35^{\prime \prime} \mathrm{O}$ & 268 \\
\hline Dendropsophus sarayacuensis & UAM-H 1487 & 2012 & $\begin{array}{l}\text { Caquetá, Belén de los Andaquíes, } \\
\text { Agua Dulce }\end{array}$ & $01^{\circ} 20^{\prime} 19.50 \mathrm{~N}, 75^{\circ} 48^{\prime} 49.35^{\prime \prime} \mathrm{O}$ & 268 \\
\hline
\end{tabular}

que los dedos y las membranas interdigitales y axilares; tienen una línea bifurcada completa en las regiones cefálica y dorsolateral que se extienden hasta la ingle y manchas en el talón con una coloración amarilla (Figura 4B); el vientre, la barbilla, la garganta y las zonas ventrales de las extremidades anteriores y posteriores son de color gris;

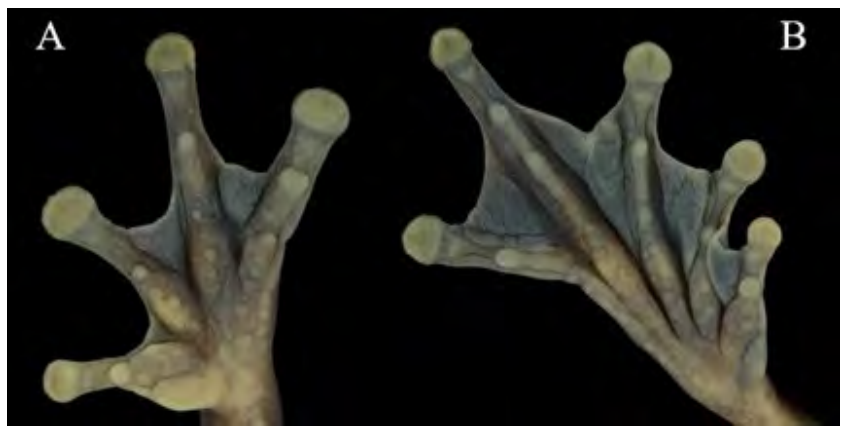

Figura 2. (A-B). Extremidad anterior izquierda con tubérculos subarticulares redondos, tubérculo palmar plano y dividido, tubérculo metacarpiano interno plano y alargado, y extremidad posterior derecha con tubérculos metatarsianos internos grandes y ovoides y tubérculos subarticulares redondos en Dendropsophus manonegra

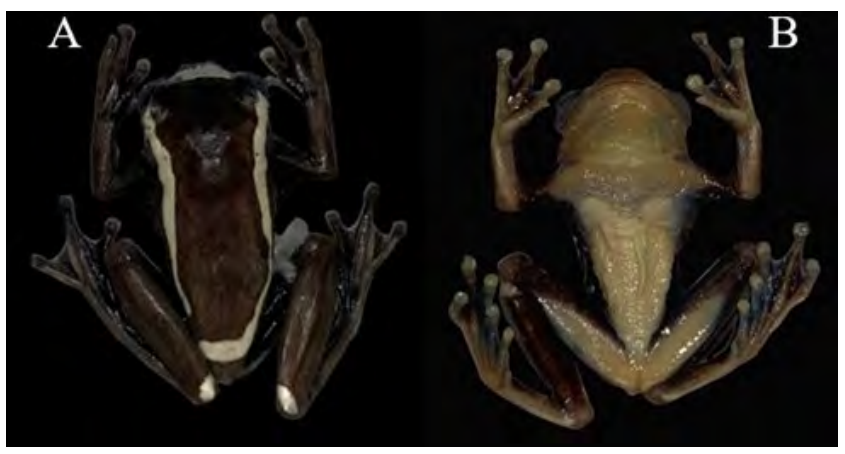

Figura 3. Vista dorsal (A) y ventral (B) de Dendropsophus manonegra. LCR 23 mm (UAM 1514 -MPO 061). Departamento de Caquetá, Colombia, Municipio de Florencia, vereda Paraíso el saco bucal es de coloración verde; presenta dos parches glandulares ubicados en la región pectoral con una coloración blanca y separados entre sí, e iris marrón cobrizo con retículas pequeñas de color marrón oscuro (Figura 4) (Rivera-Correa \& Orrico, 2013).

Descripción de los ejemplares de Dendropsophus sarayacuensis. Los especímenes presentan hocico redondeado en vista dorsal, y truncado de perfil; la longitud de los dígitos palmares se establece con la formula $\mathrm{I}<$ II $<$ III $<$ IV (Figura 5A) y la de los dígitos pediales con la formula I $<$ II $<$ V $<$ III $<$ IV (Figura 5B); las extremidades posteriores $\mathrm{y}$ el vientre son de textura granular; el dorso es liso con diminutos puntos de color negro en todo el cuerpo; presenta una coloración café claro con dos puntos de color blanco en la parte posterior del dorso y manchas de color crema con café claro sobre extremidades anteriores y posteriores (Figura 6A). Las líneas dorsolaterales de color crema comprenden desde el parpado inferior hasta el flanco medio y las líneas interoculares más anchas que largas son de color crema (Figura 6B).

Los ejemplares vivos de D. sarayacuensis tienen el dorso moteado oscuro con secciones de color café claro; presentan manchas claras que pueden ir del blanco al amarillo con bordes irregulares; la mancha interocular se extiende hasta el hocico y tiene una coloración amarilla o blanca clara, y las líneas dorsolaterales comprenden desde el parpado inferior hasta el flanco medio; las extremidades anteriores y posteriores son de color naranja; el iris es de color bronce y los machos tienen el saco bucal amarillo (Figura 7).

\section{Discusión}

Los hábitats donde se pueden encontrar estos organismos varían. Así, D. sarayacuensis se encontró en un fragmento de bosque secundario conservado de la región amazónica, a 268 m s.n.m. (Figura1), con la presencia de cuerpos de agua sin ningún tipo de alteración antrópica, coincidente con lo registrado por Cochran \& Goin (1970) y Aichinger (1987). Esta especie se ha registrado en los departamentos de Caquetá, Vaupés y Amazonas a alturas que van de los 


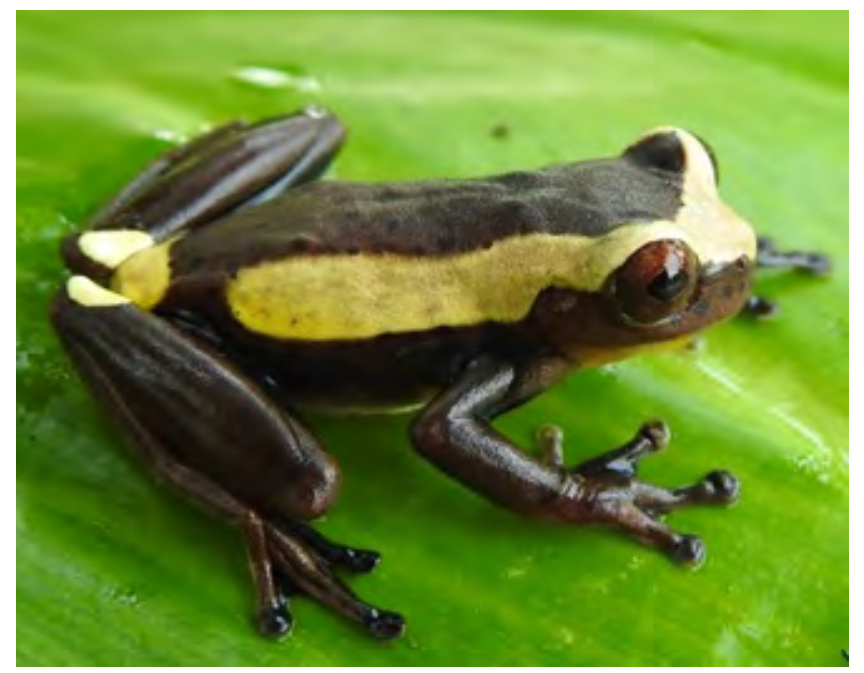

Figura 4. Vista lateral de ejemplar vivo de Dendropsophus manonegra. Departamento de Caquetá, Colombia, municipio de Florencia, vereda La Holanda. Foto: D.H. Ruiz-Valderrama, 2019

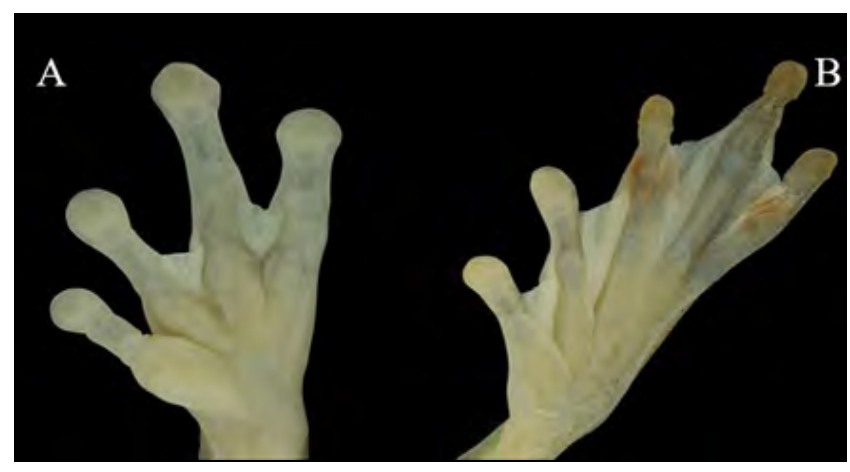

Figura 5. (A-B). Extremidad anterior izquierda con tubérculos subarticulares redondos, tubérculo metacarpiano interno plano y alargado, y extremidad posterior izquierda con tubérculos metatarsianos internos grandes y ovoides, tubérculos subarticulares redondos en Dendropsophus sarayacuensis.

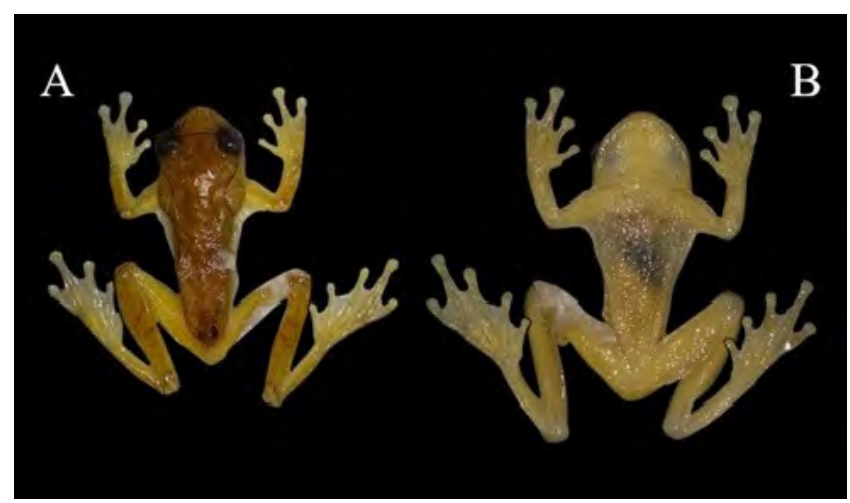

Figura 6. Vista dorsal (A) y ventral (B) de Dendropsophus sarayacuensis. LCR $18 \mathrm{~mm}$ (UAM 1486 -DHR 015). Departamento de Caquetá, Colombia, Municipio de Belén de los Andaquíes, vereda Agua Dulce, finca las Brisas.
70 hasta 210 m s.n.m. (Cochran \& Goin, 1970; Acosta, 2000; Lynch, 2005; Lynch, 2007; Lynch \& Suárez, 2011; Osorno, et al., 2011). Este nuevo registro de distribución en Caquetá demuestra que la distribución de este organismo es amplia, con variaciones altitudinales entre los 100 y los 300 m s.n.m. en esta zona del departamento, si se tiene en cuenta la distancia de los antiguos registros de distribución para la especie.

Por el contrario, D. manonegra se recolectó en zona de montaña, en cuerpos de agua sin la presencia de cobertura de dosel, similar a lo registrado por Rivera-Correa \& Orrico, 2013, pero en sitios con vegetación herbácea de la familia Juncaceae, Poaceae y Heliconeaceae (Ricaurte, et al., 2015). Las zonas donde se recolectaron estos organismos presentan una geoforma uniforme, ya que están situadas en la parte baja de una montaña, lo que permite la formación de cuerpos de agua temporales y permanentes por escorrentía y precipitación que son fundamentales para los procesos reproductivos de esta especie. Cabe resaltar que estas zonas presentan modificaciones antrópicas como la presencia de construcciones humanas, lo cual indica que D. manonegra tiene la capacidad de soportar y colonizar microhábitats modificados por la actividad antrópica.

Dendropsophus manonegra se diferencia de otras especies del complejo D. leucophyllatus por la presencia de una coloración negra azulada en sus regiones ocultas, membranas y dígitos (Rivera-Correa \& Orrico, 2013). Se considera que es la única especie con esta coloración, en tanto que D. sarayacuensis, al presentar una coloración clara, suele confundirse con otras especies como D. leucophyllatus, pero se diferencia de esta por las bandas dorsolateral delgadas que llegan hasta la mitad del cuerpo y la formación de manchas irregulares en la superficies dorsales de las extremidades (Jungfer, et al., 2010).

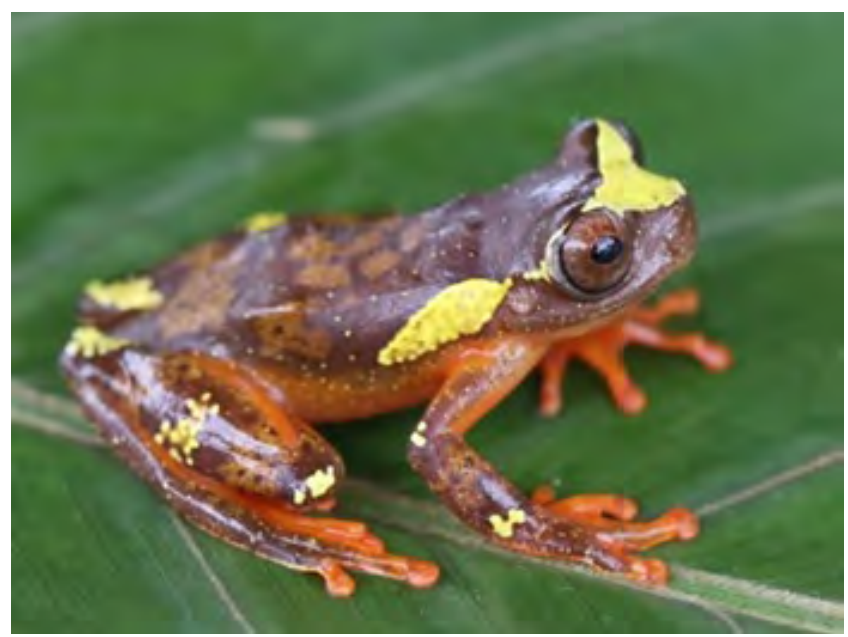

Figura 7. Vista lateral de ejemplar vivo de Dendropsophus sarayacuensis. Departamento de Caquetá, Colombia, Municipio de Belén de los Andaquíes, vereda Agua Dulce. Foto: D.H. RuizValderrama, 2019 
Los nuevos puntos de distribución para esta especie en el departamento del Caquetá tienen una distribución altitudinal que permite agruparla en el piedemonte, en alturas que comprenden desde los 400 hasta los 700 m s.n.m. con distancias significativas entre los puntos (Figura 1), lo que coincide con lo registrado por Rivera-Correa \& Orrico (2013), quienes recolectaron ejemplares desde los 740 hasta los 1.040 m s.n.m. en los departamentos de Caquetá, Cauca y Putumayo, aunque en estos dos últimos departamentos hay localidades sin precisar.

\section{Agradecimientos}

Agradecemos a la Universidad de la Amazonia, en especial al Semillero de Investigación en Herpetología - SEH, que nos facilitó la identificación y los registros de distribución; al Centro de Investigación de la Biodiversidad Andino Amazónica, INBIANAM, por el préstamo de los equipos y las instalaciones para la investigación, y al laboratorio de Entomología de la Universidad de la Amazonia, por el préstamo del equipo para la toma de fotografías macro, fundamentales para la elaboración de esta investigación.

\section{Contribución de los autores}

ANM concibió el estudio. ANM y DHRV realizaron la toma de datos en campo. Los dos autores participaron en la escritura del documento.

\section{Conflicto de intereses}

Los autores declaran que no existen conflictos de intereses con respecto al contenido y los resultados del artículo.

\section{Referencias}

Acosta-Galvis, A. R. (2019). Lista de los Anfibios de Colombia. Batrachia. Villa de Leyva. Boyaca. Colombia. Fecha de consulta: abril de 2019. Disponible en: http://research. amnh.org/herpetology/amphibia/index.html

Aichinger, M. (1987). Annual activity patterns of anurans in a seasonal Neotropical environment. Rev. Oecologia. 71 (4): 583-592.

Acosta-Galvis, A. R. (2000). Ranas, salamandras y caecilias (Tetrapoda: Amphibia) de Colombia. Rev. Biota colombiana. 1 (3): 290-319.

Caminer, M. A., Mila, B., Jansen, M., Fouquet, A., Vanegas, P. J., Chávez, G., Lougheed, S., Ron, S. R. (2017). Systematics of the Dendropsophus leucophyllatus species complex (Anura: Hylidae): Cryptic diversity and the description of two new species. Rev. PLoS one. 12 (3): 1-42.

Cochran, D. M. \& Goin, C. J. (1970). Frogs of Colombia. Edit. United States National Museum Bulletin. United States. p. 288.

Duellman, W. E., Marion, A. B., Blair, S. B. (2016). Phylogenetics, classification, and biogeography of the treefrogs (Amphibia: Anura: Arboranae). ZOOTAXA. 4104 (1): 1-109.

Faivovich, J., Haddad, C. F., Garcia, P. C., Frost, D. R., Campbell, J. A., Wheeler, W. (2005). Systematic review of the frog family hylidae, with special reference to hylinae: Phylogenetic analysis and taxonomic revision. Bulletin of the American Museum of Natural History, New York. United States. p. 240.

Frost D.R. (2019). Amphibian species of the World: An online reference. Version 6.0. American Museum of Natural History, New York. Fecha de consulta: junio de 2019. Disponible en: http://research.amnh.org/herpetology/ amphibia/index.html

Fouquet, A., Gilles, A., Vences, M., Marty, C., Blanc, M., Gemmell, N. J. (2007). Underestimation of Species Richness in Neotropical Frogs Revealed by mtDNA Analyses. PLoS one. 2 (10): 1-10.

Hödl, W. (1991). Arboreal oviposition in the Neotropical treefrogs Hyla brevifrons and Hyla sarayacuensis (Ahura: Hylidae). ÖWF. 42: 53-62.

Jungfer, K., Reichle, S. Piskurek O. (2010). Description of a new cryptic southwestern Amazonian species of leafgluingtreefrog, genus Dendropsophus (Amphibia: Anura: Hylidae). Salamandra. 46 (4): 204-213.

La Marca, E., Azevedo-Ramos, C., Coloma, L. A., Ron, S. (2004). Dendropsophus sarayacuensis. The IUCN Red List of Threatened Species 2004. Fecha de consulta: abril de 2019. Disponible en: http://dx.doi.org/10.2305/IUCN. UK.2004.RLTS.T55644A11346531

Lynch, J. D. (2005). Discovery of the richest frog fauna in the world -an exploration of the forests to the north of Leticia. Revista de la Academia Colombiana de Ciencias Exactas, Físicas y Naturales. 29 (113): 581-588.

Lynch, J. D. (2007). Anfibios. Capítulo 2, Diversidad biológica del sur de la Amazonía colombiana. p. 595-600.

Ruiz, S. L., Sánchez, E., Tabares, E., Prieto, A., Arias, J. C., Gómez, R., Castellanos, D., García, P., Rodríguez, L. (2007). Diversidad biológica y cultural del sur de la Amazonia colombiana - Diagnóstico. Corpoamazonia, Instituto Humboldt, Instituto Sinchi, UAESPNN, Bogotá D.C. p. 637.

Suárez, A. \& Lynch, J. D. (2011). Clave ilustrada de los renacuajos en las tierras bajas al oriente de los Andes con énfasis en Hylidae. Caldasia. 33 (1): 235-270.

Osorno, M., Gutiérrez-Lamus, D. L., Blanco, J. C. (2011). Anfibios en un gradiente de intervención en el noroccidente de la Amazonia colombiana. Colombia Amazónica. 4: 143-160.

Rivera-Correa, M. \& Orrico, V. G. (2013). Description and phylogenetic relationships of a new species of treefrog of the Dendropsophus leucophyllatus group (Anura: Hylidae) from the Amazon basin of Colombia and with an exceptional color pattern. ZOOTAXA. 3686 (4): 447-460.

Ricaurte, L. F., Núñez-Avellaneda, M., Pinilla, M, C., Pinilla, M. C., Marín, C. A., Velásquez-Valencia, A., Alonso, J. C., Mojica, J. I., Betancourt, B., Salazar, C., Caicedo, D., Acosta-Santos, A., Castro, W., Argüelles, J. H. (2015). Inventario y tipificación de humedales en la cuenca del río Orteguaza, Departamento del Caquetá, Amazonia Colombiana. Edit. Instituto Amazónico de Investigaciones Científicas Sinchi. p. 128.

Rodríguez, L. O. \& Duellman, W. E. (1994). Guide to the frogs of the Iquitos Region, Amazonian Perú. Natural History Museum. The University of Kansas. Kansas. United States. p. 113. 